\title{
BIBLIOTECAS, MEDIOS Y MÉTRICAS DE LA WEB SOCIAL
}

\author{
Nieves González Fernández-Villavicencio* \\ Biblioteca de Económicas de la Universidad de Sevilla
}

\begin{abstract}
Resumen: Se presenta un resumen de la tesis Rentabilidad de la biblioteca en la web social, describiendo la investigación que se ha llevado a cabo para demostrar que el uso de la web social por las bibliotecas en su oferta de productos y servicios adecuados a las expectativas de sus usuarios, es rentable para su institución al obtener diferentes tipos de ROI. Se describe la batería de indicadores y métricas para evaluar su evolución y resultados.

Palabras clave: Web social; bibliotecas; rentabilidad; servicios bibliotecarios; métricas de la web social; indicadores; ROI; indicadores predictivos.
\end{abstract}

\begin{abstract}
Title: LIBRARIES, SOCIAL MEDIA AND METRICS.
Abstract: This paper is an abstract of the dissertation Profitability of a library using social media and highlights research carried out in order to demonstrate the profitability of libraries that make use of social media both to offer products and services to the users in line with their expectations and needs and to deliver different types of ROI. A set of social media indicators to measure the evolution and results has also been established.

Keywords: Social web; libraries; profitability; library services; social media metrics; indicators; social media; ROI; return of investment; predictive indicators.
\end{abstract}

\section{INTRODUCCIÓN, CONTEXTUALIZACIÓN Y OBJETIVOS}

Este artículo presenta las principales aportaciones de la tesis doctoral Rentabilidad de la biblioteca en la web social $^{1}$, presentada por la autora en 2014 en la Universidad de Salamanca (González-Fernández-Villavicencio, 2014). La investigación responde a un conjunto de preguntas en torno a la rentabilidad del tiempo invertido en los medios sociales por parte de las bibliotecas en su oferta de productos y servicios y como forma de comunicación con los usuarios. Al mismo tiempo establece una batería de indicadores para el seguimiento de la evolución y éxito de esta actividad.

\subsection{La web social, las organizaciones y las bibliotecas}

Las empresas y organizaciones en general llevan años utilizando las herramientas, aplicaciones y servicios de la web social con distintos fines, conscientes de la oportunidad que representan los social media para establecer nuevos y profundos vínculos con los clientes/usuarios o llegar a los que no lo son, en el marco de la filosofía colaborativa y participativa de la web social y el marketing digital (Ahmed, Scheepers y Stockdale, 2014; Zare, 2014). Podemos decir que hoy día el uso de los medios sociales ha alcanzado su punto de madurez debido al uso generalizado, aunque no sin reticencias y críticas (Parry, 2012; Cefrio, 2012; Ravindran, Chua, Kuan, Goh y Lian, 2014; Isfandyari-Moghaddam y Hosseini-Shoar, 2014). Los dispositivos móviles tienen una importancia estratégica en el auge de los medios sociales y las tecnologías ponibles o el internet de las cosas, están potenciando aún más su aspecto social.

Tanto en el ámbito de las empresas como en el de las organizaciones en general, incluidas las bibliotecas, justificar el valor de lo que hacen en los medios sociales ha sido una prioridad en estos últimos años (Kingma y Mcclure, 2015; McMullen, 2013; Haddow, 2013; Oakleaf, 2011; Tessler, 2013). Buscar el ROI (Return On Investment), encontrar métricas que pongan en evidencia el retorno de la inversión realizada, se ha convertido en un objetivo en el que asociaciones, organizaciones y expertos en marketing no dejan de realizar sus apuestas y conjeturas. Los argumentos más utilizados son la inexistencia de normas que definan de forma clara y fácil cómo se obtienen esos datos, cuáles son las métricas más adecuadas para obtener la información y la falta generalizada de habilidades analíticas (Leeflang 2014; Ragan, 2013).

Cuando se aborda la medición de la rentabilidad en medios sociales se contemplan distintos tipos de ROI, desde el ROI económico, en términos financieros, hasta un beneficio en valor, traducido como Influencia, Relevancia, Reputación, el llamado IOR (Impact On Relationship) o Retorno en Relaciones. El IOR es "el valor acumulado que otorga una persona a una marca, debido al cultivo de esta relación. Mientras el ROI tiene una medida contable, el IOR es un valor. Se puede medir su impacto a través de recomendaciones, participación o lealtad" (Ted Rubin)².

*nievesg@us.es

Recibido: 26-07-2015; 2 ${ }^{\mathrm{a}}$ versión: 28-01-2016; aceptado: 03-02-2016.

GONZÁLEZ FERNÁNDEZ-VILLAVICENCIO, N. Bibliotecas, medios y métricas de la web social. Anales de Documentación, 2016, vol. 19, $\mathrm{n}^{\mathrm{o}}$ 1. Disponible en: http://dx.doi.org/10.6018/analesdoc.19.1.234001. 
Los medios sociales son fácilmente rentables para conseguir beneficios no económicos, de ahí que se hable también del SROI o ROI Social (Taladriz-Mas, 2013). Los beneficios económicos, el ROI, o el efecto ROPO (Research Online, Purchase Offline), llegan cuando obedecen a un plan de marketing digital correctamente diseñado. Los botones de compra que están presentando los medios sociales afianzan y confirman las posibilidades de retorno económico de estos medios. ${ }^{3}$

La bibliografía profesional nacional y extranjera sobre el uso de la web social por parte de las bibliotecas, ha sido abundante desde los inicios en 2006 y 2007 hasta la actualidad (Gómez Pereda y Merlo-Vega, 2010; Ferrer Martínez, 2012; Margaix-Arnal, 2013; Smeaton y Davis, 2014; McKendrick, 2013; Rogers, 2012; Ahmed, 2014; Gross, 2011; Kwanya, 2012; Singh y Gill, 2013). Todas las áreas de la biblioteca y los servicios que ofrece cada una de ellas, se ven involucrados en el uso de las herramientas y aplicaciones de la web social, que por otro lado han transformado los perfiles profesionales a la luz de los medios sociales. Áreas específicas afectadas son: creación y difusión de contenidos, gestión y dinamización de comunidades, formación interna y externa, comunidades de prácticas para la formación del personal, comunicación, gestión interna y administración, gestión de espacios, dirección, liderazgo e imagen de marca, desarrollo de planes de marketing digital, apoyo a la investigación, apoyo a la edición y publicación, apoyo a la divulgación científica, identidad digital y Atmetrics (Polger y Okamoto, 2013; Kelly, 2012).

Los beneficios que las bibliotecas pueden obtener del uso de los medios sociales quedan englobados en los cuatro grandes objetivos que toda empresa se puede plantear, a saber, aumento de las ventas, ahorro de costes, mejora de la reputación y de la satisfacción del usuario, y siempre van a depender del objetivo que se hayan propuesto. Sin objetivo no hay beneficio cuantificable.

\subsection{Coste de la inversión}

Este trabajo se basa en el concepto de rentabilidad y no en el de productividad, entendida esta última como la relación entre la cantidad de bienes y servicios producidos y la cantidad de recursos utilizados, que también puede abordarse desde el punto de vista de la productividad del personal bibliotecario. Nuestro objetivo por el contrario es averiguar si los recursos invertidos por las bibliotecas en los medios sociales están obteniendo beneficios mayores que la inversión realizada, si son rentables.

Para demostrar la rentabilidad, tendremos que aplicar la conocida fórmula "ROI= Ingresos - Gastos / Gastos x 100", por lo que veremos cual ha sido el gasto, el coste de la inversión en medios sociales por parte de las bibliotecas y cuales han sido los beneficios que se han obtenido, con el objetivo de resolver la fórmula anterior.

Para estimar el tamaño de la inversión habrá que tener en cuenta el coste de la tecnología, el coste del personal y otros costes. El coste de la inversión en medios sociales hace referencia a los costes materiales, económicos y humanos, pero también a los costes que se han evitado por el uso de estas tecnologías.

En el entorno de los medios sociales el mayor coste es la inversión hora/persona. Este dato es fácilmente identificable en el entorno de las empresas y organizaciones y podemos encontrar esta información en informes, blogs y webs principalmente (Kanter y Paine, 2012; Marco-Serrano, 2012; Ragan, 2013).

En la mayoría de los casos se habla de costes marginales exceptuando el invertido en personal y formación, es decir, el número de personas y horas de dedicación a los medios sociales y horas de formación (Primary Research Group, 2013; Polger y Okamoto, 2013; Solomon, 2013). Otras conclusiones a las que se llega en el trabajo que referenciamos relativas a las bibliotecas (González-Fernández-Villavicencio, 2014), son:

- Las empresas están formando a su propio personal como community manager, para que sea responsable de la web social y de su plan de marketing, al mismo tiempo que contratan a un consultor externo para que diseñe la estrategia. Esta es en parte la práctica que las bibliotecas han llevado a cabo hasta este momento.

- El salario que los bibliotecarios reciben por su trabajo como community manager ya sea a tiempo parcial o jornada completa de trabajo, es ligeramente inferior al establecido en el sector empresarial.

- El número de horas a la semana que las organizaciones sin ánimo de lucro o las pequeñas empresas dedican a los medios sociales es de 6 horas en un 37\% y en un $43 \%$ de los casos respectivamente. En el caso de las bibliotecas vemos como las recomendaciones y las prácticas bibliotecarias siguen la misma tónica, con un intervalo medio de horas que va desde un mínimo de 3 horas hasta un máximo de 10, con una moda de 6 horas.

- En general, los empleados consideran que la calidad de los contenidos es fundamental (potenciándose la figura del content curator), pero ven estas tareas como un aumento de la carga de trabajo.

- Los empleados prefieren que se establezcan equipos de trabajo que marquen las líneas del plan de marketing digital. 
Como parte de los resultados de esta tesis, se presentan las conclusiones obtenidas tras preguntar a un conjunto de bibliotecas que se describirán más adelante, por el número de personas con responsabilidad en los medios sociales, el número de horas semanales que le dedican, categoría profesional y tipo de formación que han recibido. Se ha realizado previamente un trabajo de búsqueda de información a través de páginas web y revistas de referencia, para obtener una media de personas y horas de dedicación en las organizaciones que utilizan los medios sociales. Destaca una publicación que aporta estos datos para el entorno de las bibliotecas (Primary Research Group, 2013). Los resultados se resumen a continuación:

- La formación ha sido mayoritariamente autodidacta (autoformación) aunque seguida muy de cerca por formación reglada por parte de la biblioteca. Algunos autores plantean la necesidad de cursos formativos en técnicas de marketing y en medios sociales (Isfandyari-Moghaddam y Hosseini-Shoar, 2014; Miret, Baró, Mañá y Velosillo, 2013).

- La media de personas con responsabilidad en los medios sociales en las bibliotecas de la muestra es de 11 . El $50 \%$ de las bibliotecas tiene una media menor de 6 . Este personal representa un $34,66 \%$ del total del personal de las bibliotecas de la muestra.

- La media de personas en las bibliotecas publicas de la muestra es muy elevado, 22,25 personas; la mitad de las bibliotecas publicas tiene menos de 13 personas con responsabilidad en medios sociales. En el caso de las universitarias, la mitad de estas bibliotecas tiene menos de 7,5 personas en los medios sociales. Las especializadas y escolares presentan valores medios muy ajustados, 3,25 y 1,5 respectivamente.

- Las bibliotecas de la muestra presentan datos muy dispersos, poco homogéneos, sobre todo en el caso de las públicas, en menor medida en las universitarias y bastante ajustados las especializadas y escolares. Dedican en líneas generales poco personal a los medios sociales ya que este tipo de responsabilidades debe ser asumida por el mayor número de personas en las organizaciones. Si nos fijamos en cada una de las bibliotecas de la muestra los datos varían desde bibliotecas con el $100 \%$ de su personal dedicado a estos medios (bibliotecas escolares y publicas) hasta otras con valores entre 5-10\%, en su mayoría universitarias.

- La media de horas semanales por persona es de 4,11, pero la desviación típica es de 3,76, por lo que tomamos como más ajustado el valor de la mediana que es de 3,7, destacando como limite superior las especializadas con una media de 7,62 horas a la semana y como inferior las públicas con una media de 3,29 horas.

Tras comparar estos resultados con los que la bibliografía ofrece relativa a empresas y organizaciones con o sin fines lucrativos, se observa coincidencia aunque con valores más bajos en el caso de las bibliotecas.

Para obtener una medida de la rentabilidad del uso de las aplicaciones de la web social en bibliotecas es imprescindible el establecimiento de un plan de marketing digital en el que se haya definido una serie de indicadores que permita una medición controlada y sistemática del ROI pero también del IOR, del impacto en las relaciones con las comunidades y el marketing de compromiso (Germano, 2011).

\subsection{Métricas de la web social}

Una vez definido el coste habrá que cuantificar los beneficios esperados estableciendo indicadores, métricas y KPIs (Key Performance Indicators) cuantitativas y cualitativas, que respondan siempre a un objetivo de negocio. Se necesita por tanto la creación de un conjunto o batería de indicadores y métricas únicas, que mida el impacto en los usuarios y la rentabilidad de la biblioteca en la web social y sirva también como análisis competitivo.

Una clasificación simple de estas métricas las divide en métricas de vanidad (vanity metrics) o métricas blandas (Marco-Serrano, 2012). Se trata de aquellos valores, - por ejemplo el número de seguidores en medios sociales-, que no llevan implícitos una acción vinculada a un resultado, como es el caso de las métricas de acción, actividad o impacto (activity metrics). La mayoría de las métricas que se ofrecen en las plataformas de medios sociales ofrecen datos cuantitativos de número de fans o seguidores que no sirven demasiado (vanity metrics), son fácilmente obtenidas pero aportan poca información. Las métricas de acción o impacto miden la actividad en relación a la marca por parte del usuario, es decir, el engagement y la interacción. Un ejemplo de ello sería el alcance por post en Facebook. Un tercer tipo de métricas son las comparativas o de análisis competitivo, que miden los resultados propios y los de la competencia. En este caso se utiliza el ratio o tasa de engagement que se encuentra en fórmulas como las de Heggestuen (2013), "Compartir la voz"4 (Share of voice) y "Compartir la conversación", en las que se pone en relación el número de seguidores por el de la competencia o las conversaciones sobre la propia empresa y sobre la competencia.

Por otro lado, las métricas pueden ser valores absolutos, sin embargo los expertos recomiendan que se utilicen valores ponderados o tasas y porcentajes ya que lo que interesa es medir la evolución del comportamiento de los usuarios en los medios sociales, si se está aproximando al objetivo establecido (Paine, 2013). 
Además de las métricas cuantitativas (seguidores, RT, comentarios, etc.), hay que establecer también métricas cualitativas, -qué se dice de la marca o nivel de satisfacción de los usuarios-, (Amec, 2013). Si las métricas de valores cuantitativos requieren de una sistematización y normalización, las métricas cualitativas son mucho más difíciles de obtener. Existen herramientas como Google alertas, SocialMention o las nubes de etiquetas a partir de los comentarios, que se utilizan con frecuencia pero que son meramente indicativas, en absoluto exhaustivas. Otros medios utilizados son las encuestas, entrevistas, grupos focales de orden cualitativo, que permiten al usuario expresar sus opiniones sobre servicios, recursos o personas. Algunas de las herramientas existentes permiten incorporar elementos cualitativos a partir de las respuestas a breves formularios por parte del usuario.

Cada vez es más abundante el número de artículos e investigaciones sobre los datos que aportan los medios sociales para detectar los sentimientos de los usuarios hacia las marcas (Ghiassi, Skinner y Zimbra, 2013) y sobre todo para hacer correlaciones y predicciones (análisis predictivo).

\subsection{Marcos y recomendaciones de organizaciones, asociaciones y expertos}

Los esfuerzos por la normalización de las métricas relativas a los medios sociales comenzaron por las empresas de relaciones públicas, pero la red está completamente plagada de iniciativas y propuestas de expertos, instituciones y empresas, que recomiendan o proponen el uso de determinadas métricas para evaluar la eficacia de los medios sociales y calcular el ROI. Destacamos la Cumbre internacional sobre medición de la actividad de comunicación y relaciones públicas celebrada en Barcelona en julio de 2010 en la que líderes como AMEC (Association for the Measurement and Evaluation of Communication) y otras asociaciones relacionadas con el marketing y las relaciones públicas realizaron una declaración de normas prácticas que sería clave en los sistemas de medición y evaluación de las relaciones públicas. A este conjunto de normas se les denomina La Declaración de Barcelona de los principios de medida, basadas en la necesidad de establecer objetivos medibles y la importancia de medir resultados de negocio. Constituye el primer paso para crear unas normas internacionales de evaluación de las relaciones públicas. ${ }^{5}$

La batería de indicadores que presentamos se basa en diferentes propuestas de empresas, organizaciones y expertos del sector del marketing digital y la comunicación, que han desarrollado marcos y cuadros de métricas en las que se está trabajando a nivel mundial, tanto en empresas con ánimo de lucro como sin él. Destaca en este ámbito la publicación de Jeffrey (2013), Eight Step Social Media Measurement Process de la AMEC. Algunas de estas organizaciones, concretamente WOMMA (Word of Mouth Marketing Association), IAB (Interactive Advertising Bureau), AMEC o IPR (Institute for Public Relations), se reunieron en 2013 en un cónclave de empresas, agencias y asociaciones para trabajar en la definición de unas normas que convencieran a todos los agentes implicados y para difundir las mejores prácticas en el campo de los medios sociales. A partir de ese año se suceden los eventos que organizan estas asociaciones y empresas para actualizar las recomendaciones y marcos de métricas, aunque hasta la fecha (mediados de 2015) no existe una única propuesta que haya reunido todos los consensos.

La experta en marketing digital Katie Paine indica en su blog ${ }^{6}$ cuáles son las características que deben tener las métricas y KPIs para que se adapten a los principios de Barcelona y a las normas del cónclave. El mensaje que se transmite es el siguiente, no se trata de cuantos likes tenga (outputs) sino de qué resultados ha obtenido la campaña (outcomes). Esto segundo es más difícil de cuantificar, las vanity metrics como índice Klout, "Me gusta" o seguidores, son esas métricas que todos quieren ver crecer. Obtener los datos precisos para establecer el ROI es costoso y hay que trabajar en una triple vía: la normalización, la analítica y el enlace con los objetivos de negocio definidos en el plan estratégico o de marketing digital. Esta situación es más complicada para aquellas organizaciones que no buscan un enriquecimiento económico, como son las organizaciones sin ánimo de lucro, aunque no imposible. En el campo de las bibliotecas, el concepto de ROI es fácilmente traducible por un aumento del uso de sus servicios, mayor demanda de sus bibliotecarios, mayor uso de las instalaciones, ahorro de costes, etc., en definitiva los objetivos que cualquier tipo de empresa aspira conseguir.

\subsection{Uso de métricas por las bibliotecas}

Algunas iniciativas que se han propuesto desde el ámbito expresamente bibliotecario, tanto asociaciones y organizaciones como expertos investigadores o bibliotecarios, son: Library Analytics and Metrics project (jiscLAMP), que fue absorbido en enero de 2015 por Jisc analytics R\&D activity ${ }^{7}$; el Grupo de Trabajo Estratégico para el Estudio del Impacto socioeconómico de las Bibliotecas en la Sociedad del Consejo de Cooperación Bibliotecaria; la Línea Estratégica 3 de REBIUN y autores como LLoret (2011), Blowers (2013), Marquina (2012), Martín Marichal (2014) entre otros.

Mención especial tienen aquellas bibliotecas españolas que ya han asumido una batería de métricas y están monitorizando su actividad en los medios sociales. Bibliotecas como la de la Universidad de Sevilla (González- 
Fernández-Villavicencio, 2007), Red de Bibliotecas Municipales de A Coruña (Maseda-Seco, 2014), Bibliotecas de la ciudad de Barcelona (Broll-Nadal, 2012), Biblioteca de la Universidad de Valencia (Aguiló, Mateu, Salom, Tomás y Trigo, 2013), FGSR (Fundación Germán Sánchez Ruiperez), (Arroyo, 2013), entre otras. Sin embargo hasta el momento, casi todas estas métricas estaban más enfocadas hacia métricas de vanidad y no existía un único cuadro de métricas que pudiera servir para realizar un análisis del contexto competitivo. Aunque sean significativas las aportaciones a este tema, concluimos que no existe un estudio que de forma amplia y sistemática haya revisado esta situación o propuesto algún sistema que midiera la rentabilidad de las actuaciones en la web social, en el ámbito de las bibliotecas españolas o no, y de carácter público.

\section{DESCRIPCIÓN DE LAS FUENTES, MATERIALES Y MÉTODOS UTILIZADOS}

Se ha realizado por un lado una revisión de la bibliografía especializada sobre el tema y un seguimiento de los términos más relacionados, a través de distintos medios especialmente Twitter.

Por otro lado, se ha llevado a cabo una serie de trabajos de campo en un conjunto de 18 bibliotecas españolas, con actividad en los medios sociales, 8 de las cuales eran universitarias (Cádiz, Zaragoza, La Laguna, Politécnica de Cataluña y Politécnica de Valencia, Carlos III, Salamanca y Sevilla), 4 públicas (Muskiz, Municipales de A Coruña, Huelva y de la ciudad de Barcelona), 4 especializadas (Instituto Cervantes, FGSR, CSIC y el Sistema Sanitario Público de Andalucía, SSPA) y 2 escolares (Leiva y Sant Jordi). Tan solo una de ellas era privada y ubicadas todas en el territorio nacional.

Se ha realizado un seguimiento de la actividad de estas bibliotecas en los medios sociales, durante un periodo de un año (2012), tiempo suficiente para comprobar la evolución de su actividad y el impacto en la comunidad, en función de una serie de parámetros o indicadores.

Para detectar los indicadores o métricas que tienen más influencia y poder de predicción sobre los objetivos ROI de cualquier tipo, se han llevado a cabo análisis de correlaciones y regresión entre las variables con la herramienta de análisis estadístico SPSS y con la ayuda de hojas de cálculo Excel.

Para la toma de datos se elaboró una plantilla con la batería de indicadores o métricas de la web social que se describe a continuación y durante ese periodo cada una de estas bibliotecas aportó mensualmente sus indicadores cuantitativos y cualitativos, en una franja entre 15 y 45, clasificados según objetivos de negocio.

\subsection{Batería de métricas que se han aplicado en este trabajo de investigación}

Se presenta el cuadro o batería de métricas e indicadores que se ha utilizado en el desarrollo de este trabajo, basado en los recomendados por empresas comerciales, organizaciones con y sin ánimo de lucro y asociaciones de prestigio en materia de marketing digital y relaciones públicas y sobre todo a partir del estudio de regresión y correlaciones sobre los datos obtenidos de las bibliotecas.

Este conjunto de métricas, indicadores y KPIs seleccionados, permite evaluar y realizar un seguimiento de la marcha del objetivo, vinculado a la estrategia de la organización. Se clasifican en una serie de áreas o categorías, es decir, en objetivos de negocio, tanto estratégicos como tácticos. Los KPIs tienen como objetivo principal medir el nivel de servicio, realizar un diagnostico de la situación, comunicar e informar sobre los objetivos, motivar a los equipos responsables del cumplimiento de los objetivos reflejados en el KPI y progresar constantemente (Ayestaran Crespo y Rangel Pérez, 2012). El número de indicadores que se suele recomendar se mueve entre 10 y 20 . Este conjunto de métricas permite ofrecer un panorama completo de la actividad en social media que está llevando a cabo la biblioteca.

Cuando hablamos de objetivos estratégicos significa que se encuentran incluidos en la planificación estratégica de la organización, es decir, que responden a los objetivos de la biblioteca a un macronivel. Para conseguirlos hace falta que se definan los objetivos tácticos, las acciones que se van a llevar a cabo. Se relaciona a continuación nuestra propuesta de objetivos estratégicos y tácticos que a su vez incluye un conjunto de métricas y KPIs. La relación completa de los indicadores utilizados en este trabajo se encuentra en este enlace por limitaciones del tamaño del artículo: <https://goo.gl/CovHbG>.

1. Visibilidad de la marca, con los objetivos tácticos:

- Alcance, popularidad, indica la audiencia a la que se llega. Se trata de una de las métricas llamadas de vanidad.

- Actividad o Frecuencia de la actividad, mide lo que la organización, en este caso la biblioteca, está haciendo en los medios sociales, su nivel de actividad. 
2. Fidelización hacia la marca, conciencia de marca, siendo el objetivo táctico el tráfico que se deriva al servicio web de referencia.

- Fidelización mide el tráfico que la organización es capaz de atraer hacia la web de referencia procedente de los medios sociales.

3. Lealtad hacia la marca, influencia y relevancia que ésta ejerce. El objetivo táctico se cubriría mediante la utilización de indicadores que midiesen la percepción social del valor de la marca.

- Influencia y relevancia mide la sensación que la marca produce en la comunidad, si es parte de la conversación en la red, cómo es percibida por sus usuarios, qué nivel de influencia ejerce en su comunidad.

4. Retorno de la inversión que se ha realizado. Ese retorno puede medirse por el poder de engagement de la marca, interacción y compromiso con la marca y por la Rentabilidad financiera. Este último se considera el objetivo de negocio más importante para las empresas y organizaciones. Para dar solución a la misma, se consideran los siguientes objetivos tácticos.

- Participación, que mide el nivel de engagement o interacción y compromiso, se trata de un objetivo de acción e impacto.

- Conversión (o ROI), que mide el número de las acciones realizadas por los usuarios potenciales (los denominados leads) que llegan a convertirse en clientes, tras realizar esas acciones que se han previsto al especificar los objetivos estratégicos y tácticos. La acción que ha de realizar el usuario es lo que se cuantifica como KPI, al estar relacionada con el objetivo especificado.

Junto a estos objetivos debemos tener en cuenta el objetivo de Reputación, que busca una rentabilidad no financiera y que se identifica como el Retorno en Influencia e IOR, es decir, el cálculo del impacto de las relaciones entre las marcas y sus seguidores. Estas relaciones se miden basándose en cuatro de los objetivos tácticos que se proponen:

- Influencia de la marca (menciones a la marca)

- El Alcance (la audiencia)

- La Participación o interacción (engagement)

- Fidelización (el tráfico a la web de referencia)

Las métricas o indicadores que se utilizan en estos últimos objetivos tácticos no indican beneficios económicos, pero sí dan una visión sobre si se ha llegado a la audiencia correcta, se ha interactuado con ella, ha aumentado en nivel de compromiso con la audiencia y si la confianza en la marca ha mejorado, es decir, su Reputación. No olvidemos que el único objetivo táctico financiero que contemplamos es el de Conversión.

Parte de los seis objetivos tácticos que aquí definimos permiten juegos de métricas cruzadas que dan lugar a diferentes porcentajes y tasas, que son los definidos como KPIs. Entre esas tasas podemos observar fundamentalmente las que tienen relación con el Engagement, que mide el nivel de interacción y participación de los usuarios dividido por el número de usuarios o la audiencia, o aquellas otras que contemplan el Contexto competitivo, es decir, comparar los datos obtenidos con los de los competidores, otras empresas u organizaciones en la misma actividad o industria.

En esta batería de indicadores se contemplan también las métricas del Sentimiento ${ }^{8}$, incluidas en los objetivos tácticos Influencia y Participación, que intentan reflejar cómo se siente la audiencia con una marca. En el objetivo táctico Influencia se incluyen los índices de influencia y las menciones a la marca y en el de Participación, los comentarios de distinto signo de los usuarios. Hay que recoger y analizar estos comentarios para detectar los sentimientos que pueden ser positivos, negativos o neutros.

En la siguiente tabla hemos reflejado cada uno de los objetivos estratégicos y sus respectivos tácticos.

Se ha incluido el objetivo estratégico de Reputación que reúne los objetivos tácticos de Alcance, Fidelización, Influencia y Participación. 


\begin{tabular}{|l|l|}
\hline Objetivos Estratégicos & Objetivos Tácticos \\
\hline VISIBILIDAD & 1.- Objetivo Alcance (Audiencia) \\
\hline VISIBILIDAD / INVERSIÓN & 2.- Objetivo Frecuencia de la actividad \\
\hline FIDELIZACIÓN & 3.- Objetivo Fidelización: tráfico a la web. \\
\hline INFLUENCIA (PERCEPCIÓN) & 4.- Objetivo Influencia: índices y menciones. \\
\hline RETORNO No ROI & $\begin{array}{l}\text { 5.- Objetivo Participación, Interacción, Engagement, } \\
\text { Influenciadores }\end{array}$ \\
\hline RETORNO ROI & 6.- Objetivo Conversión. Valor económico \\
\hline REPUTACIÓN / RETORNO No ROI & 7.- Alcance, Fidelización, Influencia y Participación \\
\hline
\end{tabular}

Tabla I. Objetivos estratégicos y tácticos.

\subsection{Metodología}

El objetivo de este trabajo ha sido por un lado conocer la actividad que este conjunto de bibliotecas ha realizado en los medios sociales durante el año 2012 y por otro, ponerla en relación con los objetivos generales de la biblioteca para valorar cuál ha sido el impacto de dicha actividad, si podemos cuantificar el retorno de la inversión realizada, si la actividad que se ha llevado a cabo ha resultado rentable. Con los resultados obtenidos se han detectado cuáles son los indicadores más frecuentes y cuales los predictores del negocio de la biblioteca en los medios sociales y se ha reelaborado la plantilla de indicadores que se presenta al final de este trabajo.

Se detallan los procesos indicados:

1. Se han descrito y analizado los datos recopilados de la actividad de estas 18 bibliotecas en los medios sociales o relacionados con ella, cuantificando su uso, observando la evolución y el comportamiento de los datos en el tiempo y realizando un benchmarking interno: estudio de la evolución de los datos a lo largo del año con un estudio estadístico descriptivo de los datos; estudio de normalidad de los datos; estudio de correlación entre los datos de cada biblioteca para determinar las posibles relaciones y dependencias entre las distintas variables e indicadores y cuando los resultados lo han permitido, estudios de rectas de regresión entre determinadas variables que hayan correlacionado de forma más significativa, con el fin de detectar consecuencias en el objetivo de negocio Conversión, a partir de la evolución de las variables de los demás objetivos de negocio.

2. Se han realizado estudios de correlaciones y rectas de regresión entre las variables y sus objetivos de negocio para determinar las posibles relaciones y dependencias entre las distintas variables e indicadores de los objetivos que recogen los datos aportados por las bibliotecas. Es decir, detectar y cuantificar el nivel de relación que pueda existir entre la inversión realizada en medios sociales y los beneficios que se pueden obtener a nivel general. Estos estudios se han llevado a cabo sobre los siguientes objetivos:

- Mayor uso de la biblioteca: objetivo Conversión. Comprobar la existencia de correlación o algún nivel de predicción entre los objetivos de negocio que se han valorado en este trabajo y el objetivo Conversión.

- Mejora de la Reputación de la biblioteca (IOR). Comprobar si existe correlación o algún nivel de predicción entre estos objetivos entre si -objetivos de Alcance, Influencia, Participación y Fidelización-. El cálculo del impacto de las relaciones entre las marcas y sus seguidores (Castelló-Martínez, 2012), se cuantifica en base a cuatro variables:

a) Alcance de la marca en los medios sociales (en base al número de seguidores de la marca en sus perfiles sociales y a su evolución);

b) la Influencia del contenido, la autoridad del contenido de la marca (menciones de la marca en la web social, enlaces al contenido de la marca, etc.);

c) la Participación e interacción de los seguidores en los perfiles de la marca;

d) la Fidelización que mide las variables del tráfico generado por el contenido de la marca en los medios sociales hacia la página web.

- Detectar si existe nivel de dependencia entre el objetivo Frecuencia de la actividad y los objetivos que indican la mejora de la Reputación de la biblioteca: Alcance, Influencia, Relevancia y Fidelización.

- Identificar cualquier otro tipo de dependencia significativa entre las variables o sumatorio de variables entre si. 
3. Se han llevado a cabo estudios de comparación, benchmarking externo, entre los resultados de los objetivos de negocio e indicadores de las 18 bibliotecas que forman parte de este estudio, para poder extraer conclusiones más generales, incluso por tipo de bibliotecas.

Con estos estudios se ha buscado identificar los indicadores más predictivos para que formen parte de la batería de métricas que se propone al final del trabajo. Un resumen de los mismos se muestra en la Tabla II.

\section{EXPOSICIÓN DE LOS RESULTADOS Y DISCUSIÓN}

La recopilación de datos producto de la actividad de las bibliotecas, ha servido para estudiar la evolución temporal de cada uno de los objetivos de negocio y sus respectivos indicadores. De esta forma se ha obtenido un mapa de la evolución de la actividad de la biblioteca en los medios sociales, sus tendencias e impacto en la comunidad y en el negocio de la biblioteca, objetivo Conversión. Además de esta información diacrónica, longitudinal, con datos recopilados durante un año, gracias a técnicas estadísticas de correlación y regresión se han podido comprobar y demostrar las reglas de dependencia que existen entre los indicadores y sus objetivos de negocio, de tal manera que podemos encontrar entre las variables predictoras, cualquiera de los indicadores analizados y entre las variables dependientes se encuentran en gran medida las variables de Conversión. Esto significa que, aunque no haya sido posible establecer un modelo matemático que pueda predecir los resultados, sí se han evidenciado aproximaciones mediante las cuales se deduce que las actividades realizadas en los medios sociales, pueden tener un impacto positivo en el negocio de la biblioteca. Las tablas con las correlaciones entre los datos de cada una de las bibliotecas, se encuentran en el siguiente enlace $<$ https:/goo.gl/qvb2Vt>. En la siguiente Tabla II se muestra un resumen de los indicadores con mayor poder de predicción, según cada objetivo de negocio.

\begin{tabular}{|c|c|}
\hline $\begin{array}{l}\text { Objetivo } \\
\text { de negocio }\end{array}$ & Indicadores más predictivos y dependientes \\
\hline$\frac{\mathscr{U}}{\stackrel{\Xi}{\Xi}}$ & $\begin{array}{l}\text { Suelen tener relaciones de dependencia entre ellas y a su vez influyen en variables de } \\
\text { Participación, en conseguir engagement e interacción con los usuarios. } \\
\text { Tienen también poder de llamamiento sobre visitas a la web de la biblioteca y se han visto } \\
\text { dependencias con variables de Conversión, Cursos de formación, Inscritos y Personas formadas o } \\
\text { Renovaciones. } \\
\text { Han sido tanto predictoras como dependientes, que lo son de las variables de Frecuencia de la } \\
\text { actividad; es decir, la actividad en los medios sociales va a influir en el tamaño de la audiencia. }\end{array}$ \\
\hline 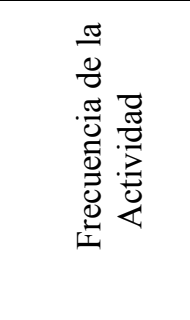 & $\begin{array}{l}\text { Han resultado predictoras en su mayoría, pero no son dependientes, ya que los factores que } \\
\text { influyen en la Frecuencia de la actividad son externos. Las más predictoras han sido entradas en SM } \\
\text { y comentarios respondidos, pero también ítems subidos o posts en blogs. } \\
\text { Las variables de Frecuencia de la actividad son predictoras de variables de Conversión y de } \\
\text { Participación, así como de Alcance. Son predictoras de variables relacionadas con la formación y el } \\
\text { préstamo archivos descargados e incluso PI. Por ejemplo, posts en blogs es predictora y dependiente } \\
\text { de variables de Conversión, influye en un mayor uso de la biblioteca, así como chats atendidos que es } \\
\text { también predictora de variables de Conversión. }\end{array}$ \\
\hline 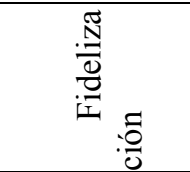 & $\begin{array}{l}\text { Una parte de estas variables son tanto predictoras como dependientes. } \\
\text { Al ser variables relacionadas con la web, mantienen dependencias y son predictoras de las demás } \\
\text { variables web como las de Conversión, pero también lo son del resto de los objetivos de negocio, } \\
\text { Alcance, Frecuencia, Influencia y Participación. }\end{array}$ \\
\hline 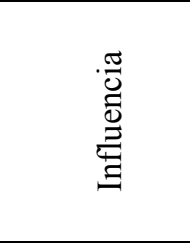 & $\begin{array}{l}\text { Estas variables deben ser predictoras de variables de Participación, ya que constituyen el paso } \\
\text { anterior a la interacción del usuario. } \\
\text { Menciones es la variable que más predice y más dependiente es de variables de todos los } \\
\text { objetivos de negocio, pero a su vez es predictora de varias variables de Participación, como se ve en } \\
\text { el resto de las variables de Influencia. } \\
\text { Los índices de Influencia no son dependientes de las variables. }\end{array}$ \\
\hline 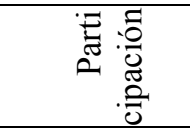 & $\begin{array}{l}\text { Son predictoras, pero sobre todo dependientes, hasta el caso de que muchas de las variables han } \\
\text { resultado dependientes de variables de todos los objetivos. Predictoras sobre todo de Conversión, } \\
\text { pero también de su mismo objetivo de Participación. }\end{array}$ \\
\hline 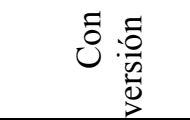 & $\begin{array}{l}\text { Son las variables más predictoras y más dependientes, en muchos casos, como préstamos, PI, de } \\
\text { variables de todos los objetivos. Han resultado predictoras y dependientes en muchos casos de las } \\
\text { variables de su mismo objetivo de Conversión. }\end{array}$ \\
\hline
\end{tabular}

Tabla II. Indicadores más predictivos y dependientes por objetivos de negocio. 
Además del estudio por biblioteca o diacrónico, el realizado a los indicadores y sus objetivos de negocio se ha abordado desde la perspectiva comparativa, sincrónica o transversal, por objetivo de negocio e indicador, para ver la forma en la que han reaccionado las bibliotecas, cual ha sido su comportamiento, incluso por tipos de bibliotecas. De esta forma se ha hecho una radiografía a cada indicador, a aquellos que han sido más usados por las bibliotecas de la muestra, con suficiente número de incidencias para llegar a ser representativos. Los resultados de este enfoque permiten que las bibliotecas puedan compararse entre sí al haber extraído de este estudio valores medios representativos de la población. Por otro lado no es difícil encontrar en la bibliografía y en la red, ejemplos de estas métricas en organizaciones y servicios, que pueden servir también como referentes de buenas prácticas.

Como resultado, tras el estudio de correlaciones y variables predictoras y más frecuentes, se ha elaborado un cuadro de métricas e indicadores y contempla tanto los indicadores clave para medir la evolución de los datos en cada biblioteca y compararse con otras, como los indicadores específicos para cada medio social.

Debido a su naturaleza cambiante, los medios sociales están en continua evolución generando nuevas funcionalidades y adaptaciones a las necesidades, exigencias y cambios de comportamiento de los usuarios o como producto de nuevas investigaciones en la audiencia, pero sobre todo se deben a los intereses comerciales de los proveedores de estos medios. Este cambio constante lleva consigo la aparición y desaparición de medios, funcionalidades e indicadores. Por esta razón, los indicadores clave que se aportan contemplan unas características que les permiten adaptarse a cualquier canal o medio social en los que la biblioteca haya decidido desarrollar su actividad. Deben responder a una serie de cuestiones como: quién es la audiencia, qué actividad se ha llevado a cabo, cuál ha sido la respuesta a esa actividad por parte de los usuarios y qué resultados se han obtenido.

Por otro lado, el estudio de campo se realizó en 2012 y las bibliotecas que participaron en el mismo destacaban por el uso de estos medios, esto parece indicar que con la madurez alcanzada, la situación de estas bibliotecas está hoy día más generalizada y los resultados de este estudio puedan ser extrapolables a la mayoría.

\subsection{Plantilla con la batería de indicadores en social media}

Ofrecemos el cuadro o batería de indicadores resultante del estudio que se ha llevado a cabo sobre los resultados obtenidos en las bibliotecas de la muestra y que consideramos adecuados para medir tanto la evolución de la actividad de la biblioteca en los medios sociales y su comparación con otros servicios del mismos sector, como para su integración en un plan de marketing o campaña promocional, en función de los objetivos que se hayan establecido. Esta plantilla tiene una versión disponible online accesible en Google Drive <https://goo.gl/WPQzZz>.

En la plantilla con la batería de indicadores se describe cada uno de estos objetivos tácticos y los indicadores que incluye. Hay que tener en cuenta que las propias herramientas van añadiendo nuevos indicadores y estadísticas sobre los mismos y por otro lado, no cesan de salir nuevas herramientas y características que medir. Así mismo, y tras un período de evaluación de datos, es posible que indicadores que no han sido tenidos en cuenta en este momento aumenten su relevancia al variar las posibilidades de contenido que ofrece cada herramienta.

Además de los valores absolutos que requiere cada métrica se han añadido las variables sumatorias y los porcentajes de crecimiento intermensual, es decir, los valores en porcentajes o tasas que podrían recogerse dentro de cada objetivo táctico. Se añaden también las herramientas y servicios de medición, necesarios para el proceso de toma de datos y se presenta el mapa mental de herramientas para monitorizar, que la autora de esta investigación ha realizado en Mindomo 9 .

Se presenta la plantilla de indicadores para medir la rentabilidad en los medios sociales. Esta plantilla se compone de una serie de cuadros en los que hemos contemplado tanto las métricas generales y las métricas clave como aquellas específicas para un medio social concreto.

\subsubsection{Cuadro general de metadatos}

El cuadro general considera todas las métricas desde el punto de vista de sus especificaciones generales o metadatos y afecta a cualquier tipo de indicador que quiera usarse, actual o futuro, en la medición de la rentabilidad. Se contempla en primer lugar cada uno de los objetivos de negocio y por cada uno de ellos se añade la finalidad del objetivo en sí y en relación con las métricas, el tipo de métrica o indicador habitual que se utiliza -si son valores absolutos o tasas-, el valor que aporta la métrica, los indicadores para cada uno de los objetivos, las fórmulas que se pueden utilizar para obtener los indicadores y las herramientas que se recomiendan para su toma de datos. Se hace referencia al mapa en Mindomo. 


\subsubsection{Cuadro de métricas clave}

El segundo de los cuadros son las métricas clave, una selección de las que se han estudiado en esta investigación ya sea por su mayor frecuencia entre las bibliotecas y carácter predictivo o bien por la importancia estratégica de la información que ofrece. Se trata de aquellas métricas que por otro lado consideramos imprescindibles para realizar un estudio diacrónico de la evolución de los datos por bibliotecas y para compararse con la competencia.

\subsubsection{Batería de indicadores por medio social}

Los cuadros específicos por medio social tienen como finalidad recopilar cada uno de los indicadores o métricas que ofrecen tanto los propios medios como herramientas específicas para su gestión. En función del plan de marketing o campaña de promoción, se seleccionarán aquellos que sean más adecuados para el objetivo que se haya definido.

\section{CONCLUSIONES O RECOMENDACIONES}

En este trabajo se presenta un estudio descriptivo y análisis de correlaciones y dependencias, rectas de regresión y variables predictoras, de los indicadores de uso de la web social y métricas tradicionales en el conjunto de las 18 bibliotecas de la muestra, tomados durante el año 2012 y clasificados en función de los objetivos de negocio de cada una de las bibliotecas: Conversión, Alcance de los seguidores, Fidelización o tráfico a la web, Influencia de la marca, Participación y Frecuencia de la actividad de la biblioteca en los medios sociales.

A partir de los resultados obtenidos, podemos afirmar que existe una dependencia entre las variables, tanto con el objetivo de Conversión como aquellos que hacen referencia a los objetivos relacionados con reputación, que son el resto de los objetivos que se han enumerado. Aplicado este razonamiento a nuestro entorno nos serviría para demostrar a las personas responsables de que el tiempo y recursos invertidos han servido para conseguir los objetivos de la empresa, ya que se puede mostrar la evolución de los KPI; y al mismo tiempo, tenemos la oportunidad de verificar si nuestra estrategia funciona, si es necesario realizar cambios y si hemos estructurado correctamente los protocolos.

De cualquier forma es importante que no pensemos exclusivamente en indicadores concretos de medios sociales, sino en qué es lo que queremos medir y cuál es la información crítica y relevante que hay que conseguir. La selección de indicadores que se haga tiene que responder a una serie de cuestiones fundamentales e imprescindibles para una correcta y completa evaluación de la actividad:

1. Cuál es la audiencia, el tamaño de la comunidad a la que le puede llegar el mensaje de la biblioteca. Los indicadores son aquellos que miden a los seguidores en cada medio social que se mantenga y cualquier otro tipo de audiencia en medios sociales que puedan aparecer.

2. Cuál ha sido la actividad de la marca en estos medios. Este dato va a depender del medio y serán tanto originales como recomendaciones de contenidos originales de los demás. La calidad de lo que se aporta es importante así como la frecuencia y adecuación a las necesidades de los usuarios y cada vez más, el tiempo que se retenga al usuario. Se incluyen aquí las tareas relacionadas con los gestores de contenidos, pero también los de comunidad ya que además de los contenidos de calidad y de interés para los colectivos concretos, tiene que buscarse la conversación con los usuarios, el diálogo. Para ello se responde a comentarios, se plantean preguntas o sugerencias en los espacios propios y ajenos, o se convocan eventos.

3. Cómo ha sido la respuesta de la audiencia a los mensajes de la marca biblioteca, qué nivel de interacción ha conseguido en los medios sociales que ha utilizado, cuánto tiempo se ha permanecido en los medios sociales de la marca. Para ello se deben utilizar los indicadores que ofrece cada plataforma para medir ese nivel de interacción: comentarios, compartidos, "Me gusta", tasas de participación de interacción, etc.

4. Qué resultados se han conseguido, si se ha alcanzado el objetivo propuesto. Este resultado se mide tanto a través del tráfico a la sede/sedes web como en el uso de la biblioteca y va a depender del objetivo que se haya marcado.

\section{NOTAS}

${ }^{1}$ Una segunda parte de la tesis relativa al plan de marketing ha sido presentada ya en forma de artículo: ROI en los medios sociales: campañas de marketing y bibliotecas. El Profesional de la Información, vol. 24, nº 1. http://dx.doi.org/10.3145/epi.2015.ene.03.

2 Ted Rubin, autor de Return on Relationship. Más información en: < http://tedrubin.com/return-on- relationship-the-new-measure-of-success/>.

3 Artículo de Rosa Jiménez Cano en El País "Comprar, el botón que invade la red" $<$ http://economia.elpais.com/economia/2015/06/05/actualidad/1433494382_425544.html>.

$4<$ http://www.socialmediaexaminer.com/metrics-to-track-your-social-media-efforts/>.

5 Acceso a los contenidos en la web de AMEC <http://amecorg.com/important-stuff/ $>$. 
$6<$ http://painepublishing.com/measurementadvisor/12-signs-that-your-measurement-program-is-not-in-compliance-with-the-barcelona-principlesand-how-to-fix-them-2/>.

${ }^{7}<$ http://analytics.jiscinvolve.org/wp/>.

8 Ibídem.

9 Mapa mental de Mindomo sobre "Herramientas de evaluación y monitorización en la web social", que se encuentra disponible en la aplicación Mindomo $<$ https://goo.gl/sjNMCo $>$.

\section{BIBLIOGRAFÍA}

AGUILÓ, P. et al. Tejiendo la red: modelo 2.0 de las bibliotecas de la Universidad de Valencia. XII Workshop de Rebiun sobre Proyectos Digitales. Lleida, $2013 . \quad$ Disponible en: $<$ http://roderic.uv.es/bitstream/handle/10550/30475/tejiendo_la_red.pdf?sequence=1 $>$ [Consulta: 28 de julio de 2015].

AHMED, A.; SCHEEPERS, H. y STOCKDALE, R. Social Media Research: A Review of Academic Research and Future Research Directions. Pacific Asia Journal of the Association for Information Systems, 2014, vol. 6, $\mathrm{n}^{\mathrm{o}}$ 1, $\mathrm{p}$. 3. Disponible en: $<$ http://aisel.aisnet.org/pajais/vol6/iss1/3> [Consulta: 22 de julio de 2015].

AMEC.5a Cumbre para la medición y la evaluación de la Comunicación, 2013. Disponible en: $<$ http://amecinternationalsummit.org/ $>$ [Consulta: 23 de julio de 2015].

ARROYO-VÁZQUEZ, N. Medir el trabajo en medios sociales: la experiencia de la Fundación Germán Sánchez Ruipérez. XIII Jornadas Españolas de Documentación Fesabid 2013. Disponible en: $<$ http://www.comunidadbaratz.com/blog/actas-de-las-xiii-jornadas-espanolas-de-documentacion-fesabid13> [Consulta: 23 de julio de 2015].

AYESTARÁN CRESPO, R. y RANGEL PÉREZ, C. Planificación estratégica y gestión de la Publicidad. ESIC, 2012.

BLOWERS, H. Measuring Social Media and the Greater Digital Landscape. Computers in Libraries, 2013, vol. 32, $\mathrm{n}^{\circ}$ 7. $\quad<$ http://www.infotoday.com/cilmag/sep12/Blowers--Measuring-Social-Media-and-the-Greater-DigitalLandscape.shtml $>$ [Consulta: 22 de julio de 2015].

BRÖLL-NADAL, A.; GÁNDARA-SANZ, A. y CABRÉ-SERRA, D. Comunicar en la Web. Estrategias de Biblioteques de Barcelona. El Profesional de la Información, 2012, vol. 21, n ${ }^{\circ}$ 4, p. 406-412. http://dx.doi.org/10.3145/epi.2012.jul.13.

CASTELLÓ-MARTINEZ, A. El estudio del retorno de la inversión y el impacto en la relación de la comunicación empresarial y publicitaria en plataformas sociales: herramientas disponibles en el mercado. Actas del 2o Congreso Nacional sobre Metodología de la Investigación en Comunicación. 2013, S.1., s.n., p. 411-427. Disponible en: $<$ http://rua.ua.es/dspace/bitstream/10045/28195/1/AE- IC_Segovia_Araceli_Castello.pdf $>$ [Consulta: 22 de julio de 2015].

CEFRIO. Gérer les enjeux et risques juridiques du Web 2.0. Disponible en: $<$ http://www.cefrio.qc.ca/media/uploader/guide_juridique_Web2.pdf $>$ [Consulta: 22 de julio de 2015].

FERRER MARTÍNEZ, F.J. El paper de les biblioteques en el web 2.0. S.1., s.n. (Proyecto fin de carrera). Universidad Politécnica de Valencia, 2012. Disponible en: <http://riunet.upv.es/handle/10251/17138> [Consulta: 22 de julio de 2015].

GERMANO, M. The library value deficit. The Bottom Line: Managing Library Finances, 2011, vol. 24, $\mathrm{n}^{\circ} 2$, p. $100-$ 106. http:// dx.doi.org/10.1108/08880451111169124.

GHIASSI, M.; SKINNER, J. y ZIMBRA, D. Twitter Brand Sentiment Analysis: A hybrid system using n-gram analysis and Dynamic Artificial Neural Network. Expert Systems with Applications, 2013, vol. 40, $\mathrm{n}^{\mathrm{o}} 16$. http://dx.doi.org/10.1016/j.eswa.2013.05.057.

GÓMEZ PEREDA, N. y MERLO VEGA, J.A. Experiencias bibliotecarias con las tecnologías sociales. Educación y Biblioteca, 2010, no 177 (mayo-junio). Disponible en: <http:/www.baratz.es/portals/0/noticias/Dossier Educación y Biblioteca.pdf $>$ [Consulta: 22 de julio de 2015].

GONZÁLEZ-FERNÁNDEZ-VILLAVICENCIO, N. Biblioteca 2.0 en España (el camino recorrido). Boletín de la Asociación Andaluza de Bibliotecarios, 2007, año 22. $\mathrm{n}^{\circ}$ 86-87 (enero-junio), p. 29-46 Disponible en: $<$ http://dialnet.unirioja.es/descarga/articulo/2544094.pdf $>$ [Consulta: 22 de julio de 2015].

GONZÁLEZ-FERNÁNDEZ-VILLAVICENCIO, N. La rentabilidad de la biblioteca en la web social. Tesis doctoral presentada en la Universidad de Salamanca, 2014. Disponible en: <http://hdl.handle.net/10366/125114> [Consulta: 20 de julio de 2015].

GROSS, M. Gestión del Conocimiento: La figura de los curadores de contenidos contra la infoxicación, 2011. Disponible en: <http://manuelgross.bligoo.com/20110915-gestion-del-conocimiento-la-figura-de-los-curadores-decontenidos-contra-la-infoxicacion $>$ [Consulta: 22 de julio de 2015]. 
HADDOW, G. Academic library use and student retention: A quantitative analysis. Library \& Information Science Research, 2013, vol. 35, n 2, p. 127-136. http://doi.org/10.1016/j.lisr.2012.12.002.

HEGGESTUEN, J. The Decline Of Social ROI - How Social Media Marketers Are Moving Towards More Basic, No- Frills Metrics. BI Intelligence, 2013, vol. Sept. Disponible en: <http://www.bullfax.com/?q=node-declinesocial-roi-\%E2\%80\%94-how-social-media-marketers-are-mov> [Consulta: 22 de julio de 2015].

ISFANDYARI-MOGHADDAM, A. y HOSSEINI-SHOAR, M. Factors affecting Web 2.0 adoption: a case study. Program: electronic library and information systems, 2014, vol. $48, \quad \mathrm{n}^{\mathrm{o}} \quad 1, \quad \mathrm{p}$. $2-15$. http://dx.doi.org/10.1108/PROG-02-2012-0005.

JEFFREY, A. Social Media Measurement: A Step - by - Step Approach Using the AMEC Valid Metrics Framework. IPR Institute for Public Relations, 2013, $\mathrm{n}^{\mathrm{o}}$ junio. Disponible en: < http://www.instituteforpr.org/topics/socialmedia-measurement-a-step-by-step- approach/> [Consulta: 22 de julio de 2015].

KANTER, B. y PAINE, K.D. Measuring the Networked Nonprofit: Using Data to Change the World. San Francisco: John Wiley \& sons, 2012.

KELLY, B. What next for Libraries? Making sense of the future. Emerging Technologies in Academic Libraries 2012: International Conference, Trondheim. Disponible en: $<$ http://opus.bath.ac.uk/31642/> [Consulta: 22 de julio de 2015].

KINGMA, B. y MCCLURE, K. Lib-Value: Values, Outcomes, and Return on Investment of Academic Libraries, Phase III: ROI of the Syracuse University Library. College \& Research Libraries Pre-print, 2015. http://dx.doi.org/10.5860/crl.76.1.63.

KWANYA, T.; STILWELL, C. y UNDERWOOD, P.G. Intelligent libraries and apomediators: Distinguishing between Library 3.0 and Library 2.0. Journal of Librarianship and Information Science, 2012, vol. 45, $\mathrm{n}^{\mathrm{o}} 3, \mathrm{p}$. 187-197. Disponible en: <http://lis.sagepub.com/cgi/doi/10.1177/0961000611435256> [Consulta: 22 de julio de 2015].

LEEFLANG, P.S.H.; VERHOEF, P.C.; DAHLSTRÖM, P. y FREUNDT, T. Challenges and solutions for marketing in a digital era. European Management Journal, 2014, vol. $32, \quad \mathrm{n}^{\circ} \quad 1, \quad$ p. $1-12$. http://dx.doi.org/10.1016/j.emj.2013.12.001.

LLORET-ROMERO, N. ROI. Measuring the social media return on investment in a library. The Bottom Line: Managing Library Finances, 2011, vol. 24, nº 2, p. 145-151. http://dx.doi.org/10.1108/08880451111169223.

MARCO-SERRANO, F. Qué es el Social Media ROI y cómo medirlo. Social MediaBlog.es, 2012. Disponible en: $<$ http://www.socialancer.com/guia-ebook-social-media-roi/> [Consulta: 22 de julio de 2015].

MARGAIX-ARNAL, D. Los social media en las bibliotecas universitarias españolas: su presencia y las guías de usos y estilo (TFG), Universidad de León. Grado en Información y Documentación, 2013. Disponible en: $<$ https://buleria.unileon.es/handle/10612/2847> [Consulta: 22 de julio de 2015].

MARQUINA-ARENAS, J. Plan social media y community manager. Barcelona: Editorial UOC, 2013. ISBN 8490292396.

MARTÍN MARICHAL, C. Las bibliotecas universitarias en las redes sociales. Principales cifras 2014. Rebiun. 2014. Disponible $<$ http://www.rebiun.org/documentos/Documents/IIIPE_2020_LINEA3/IIIPE_Linea3_bibliotecas_universitarias_re des\%20sociales_REBIUN_2014.pdf $>$ [Consulta: 22 de julio de 2015].

MASEDA-SECO, $\overline{\mathrm{D}}$. Grupo de trabajo web 2.0 en las Bibliotecas Municipais da Coruña: un engranaje sofisticado que crea comunidad, genera, comparte y difunde contenido en la WWW. 2014. Disponible en: $<$ http://eprints.rclis.org/22571/> [Consulta: 22 de julio de 2015].

MCKENDRICK, J. Libraries: At the Epicenter of the Digital Disruption - Academic Libraries Edition. Library Resource Guide, 2013. Disponible en: $<$ http://www.libraryresource.com/SectorReports/Details.aspx?ResearchReportsID=365> [Consulta: 22 de julio de 2015].

MCMULLEN, A. The value of values. The Bottom Line, 2013, vol. 26, $\mathrm{n}^{\mathrm{o}}$ 1, p. 4-6. http://dx.doi.org/10.1108/08880451311321519.

MIRET, I.; BARÓ, M.; MAÑÀ, T. y VELOSILLO, I. Las bibliotecas escolares en España. Dinámicas 2005-2011, 2013. <http://leer.es/files/2013/09/estudio.pdf> [Consulta: 22 de julio de 2015].

OAKLEAF, M. What's the value of an academic library? The development of the ACRL value of academic libraries comprehensive research review and report. Australian Academic \& Research Libraries, 2011, vol. 42, $\mathrm{n}^{\circ}$ 1, p. 112. http://dx.doi.org/10.1080/00048623.2011.10722200.

PAINE, K.D. The Conclave. Complete social media measurement standards, 2013, junio. Disponible en: $<$ http://www.smmstandards.com/wp- content/uploads/2013/06/Complete-standards-document4.pdf $>$ [Consulta: 22 de julio de 2015].

PARRY, F. Book Review: Networks Without a Cause: A Critique of Social Media. The Electronic Library, 2012, $\mathrm{n}^{\circ}$ 4, p. 1-2. 
POLGER, M.A. y OKAMOTO, K. Who's Spinning the Library? Responsibilities of Academic Librarians who Promote Article. Library Management, 2013, vol. 34, $\mathrm{n}^{\mathrm{o}} \quad 3, \quad$ p. $236-253$. http://dx.doi.org/10.1108/01435121311310914.

PRIMARY RESEARCH GROUP. Academic Library Reputation Management Practices. 2013. ISBN 9781574402643.

RAGAN. PR Measurement. Lawrence Ragan Communications. S.1., 2013. Disponible en: $<$ http://kdpaine.blogs.com/files/ragan_prmeasurement_whitepaper_v3-2.pdf $>$ [Consulta: 22 de julio de 2015].

RAVINDRAN, T. et al. Antecedents and Effects of Social Network Fatigue. Journal of the Association for Information Science and Technology, 2014. http://dx.doi.org/10.1002/asi.23122.

ROGERS, C.R. Social Media, Libraries, and Web 2.0: How American Libraries are Using New Tools for Public Relations and to Attract New Users - Fourth Annual Survey November 2011, 2012, $\mathrm{n}^{\mathrm{o}}$ noviembre. Disponible en: $<$ http://dc.statelibrary.sc.gov/bitstream/handle/10827/7271/SCSL_Social_Media_Libraries_2011.pdf $>$ [Consulta: 22 de julio de 2015].

SINGH, K.P. y GILL, M.S. Web 2.0 technologies in libraries: a survey of periodical literature published by Emerald. Library Review, 2013, vol. 62, nº 3, p. 177-198. http://dx.doi.org/10.1108/00242531311329491.

SMEATON, K. y DAVIS, K. Social technologies in public libraries: exploring best practice. Library Management, 2014, vol. 35, no 3, p. 224-238. http://dx.doi.org/10.1108/lm-09-2013-0087.

SOLOMON, L. The librarian’s nitty-gritty Guide to social media. Chicago: ALA, 2013.

TALADRIZ-MAS, M. Los servicios de información y el retorno de la inversión: cómo llegar a conocerlo. El Profesional de la Información, 2013, vol. 22, nº 4, p. 281-285. http://dx.doi.org/10.3145/epi.2013.jul.01.

TESSLER, A. Economic valuation of the British Library. January 2013. A report for the British Library [en línea]. Disponible en: <http:/www.bl.uk/aboutus/stratpolprog/increasingvalue/britishlibrary_economicevaluation.pdf> [Consulta: 22 de julio de 2015].

ZARE, A. y ASGARY, S. A Review for the Online Social Networks Literature (2005-2011). European Journal of Business and Management, 2014, vol. 6, n 4, p. 22-37. 